\title{
Clinical importance of HER2 immunohistologic heterogeneous expression in core-needle biopsies vs resection specimens for equivocal (immunohistochemical score $2+$ ) cases
}

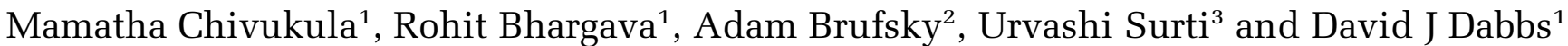 \\ ${ }^{1}$ Department of Pathology, Magee Women's Hospital of UPMC, Pittsburgh, PA, USA; ${ }^{2}$ Department of Medical \\ Oncology, Breast Cancer Care Center, Magee Women's Hospital of UPMC, Pittsburgh, PA, USA and \\ ${ }^{3}$ Department of Cytogenetics Magee, Women's Hospital of UPMC, Pittsburgh, PA, USA
}

\begin{abstract}
HER2 oncoprotein is overexpressed in 15-20\% of breast carcinomas and is associated with poor outcome. The $2+$ group is considered equivocal, since gene amplification is observed in some but not others. The aim of our study is to ascertain if there is clinical significance to heterogeneity of HER2 immunohistologic expression in breast core-needle biopsies vs surgical resection specimens. A total of 37 invasive breast carcinomas diagnosed on core-needle biopsies and scored $2+$ by HER2 immunohistochemical assay were selected from our files. The results were obtained on these selected cases, of which 19 cases were nonamplified and 18 cases were amplified. The follow-up resection specimens were reviewed and two additional tumor blocks were selected in each case for HER2 immunostaining. The 74 tissue blocks were examined for HER2 using antibody clone CB11 on the Benchmark XT and scored as negative (score 0 or $1+$ ), weakly positive $(2+)$ or strongly positive $(3+)$. Results within the amplified group, $56 \%(11 / 18)$ showed significant areas with $3+$ score in both blocks, $28 \%(5 / 18)$ remained as $2+, 11 \%(2 / 18)$ showed score $0-1+$. In the nonamplified group, $42 \%(8 / 19)$ had score $0-1+, 37 \%(7 / 19)$ remained as $2+, 0 \%(0 / 19)$ had score $3+$. Five (5) cases showed heterogeneous staining in both the groups. In the amplified group, $56 \%$ of cases showed strong $3+$ in both the blocks of which half of these cases had areas of $2+$. Fluorescence in situ hybridization was performed on a representative resection specimen block. In the amplified group $72 \%(13 / 18)$ cases were amplified, $22 \%(4 / 18)$ were nonamplified. In the nonamplified group, no amplification is detected in a great majority of cases $89 \%$ (17/19). HER2 immunohistochemistry on core-needle biopsies is usually predictive of tumor HER2 status. However, performing fluorescence in situ hybridization on core-needle biopsies almost completely resolves the issue of heterogeneous expression of HER2.

Modern Pathology (2008) 21, 363-368; doi:10.1038/modpathol.3801021; published online 1 February 2008
\end{abstract}

Keywords: HER2; FISH; core-needle biopsy; breast carcinoma; IHC; Herceptin

HER2 is a proto-oncogene, located on chromosome 17 and is amplified in $15-20 \%$ of breast carcinomas, which signifies a poor prognosis and predicts a poor response to hormonal therapy. ${ }^{1,2}$ American Society of Clinical Oncology and National Comprehensive Cancer Network guidelines recommend HER2 testing for all primary breast carcinomas. ${ }^{2,3}$ Assessment of HER2 status of breast carcinomas is critical for the management of advanced breast cancer. With the

Correspondence: Dr M Chivukula, MD, Department of Pathology, Magee Women's Hospital of UPMC, 300 Halket Street, Pittsburgh, PA-15213, USA.

E-mail: mchivukula@mail.magee.edu

Received 23 July 2007; revised 5 December 2007; accepted 7 December 2007; published online 1 February 2008 recent focus on the targeted therapies and latest introduction of Trastuzumab (Herceptin ${ }^{\circledR}$ ), a humanized monoclonal antibody to the extracellular domain of HER2, appears to be promising for the patients with metastatic breast carcinoma. However, due to the cardiotoxic effect of this drug, it becomes important to evaluate the HER2 status accurately and select patients who will benefit from the therapy.

HER2 proto-oncogene amplification is usually accompanied by protein overexpression. The amplification is generally determined by fluorescent in-situ hybridization (FISH) method and the protein expression is determined by immunohistochemistry. In a typical pathology laboratory, both assays are performed on formalin-fixed paraffin-embedded 
tissue material. In FISH method, a labeled probe is added to the denatured patient's DNA, which recognizes the target gene and enumerates fluorescence signals, which are counted using a fluorescence microscope to assess HER2 gene amplification. In contrast, immunohistochemistry is performed using monoclonal or polyclonal standardized antibody kits, which generates a colored reaction that is evaluated by bright field microscopy. ${ }^{4}$ American Society of Clinical Oncologists/ College of American Pathologists guideline recommendations for HER2 testing have clearly defined the categories of positive (immunohistochemistry score $3+$ ), equivocal (immunohistochemistry score $2+$ ) and negative (immunohistochemistry score $0 / 1+$ ) categories. Among these categories, the equivocal or weakly positive $(2+)$ category creates confusion about trastuzumab treatment, therefore it requires an additional FISH test as recommended by American Society of Clinical Oncologists/College of American Pathologists guidelines. ${ }^{2}$ Comparison of HER2 results by immunohistochemistry and FISH has been addressed by few studies in literature. In these studies, the greatest concordance rate is observed in immunohistochemistry score $3+$ cases in which majority showed amplification by FISH. In the equivocal $2+$ cases results from various studies show variable results with a high rate of falsepositive results is observed by follow-up negative FISH results. ${ }^{5}$ In other studies, the amplification rate of HER2 in the equivocal group is observed to be approximately $25 \%, 4,6$ thereby emphasizing the need for additional FISH testing. Core-needle biopsies have been shown to be a valid tissue sample for evaluation of prognostic markers including testing of HER2, particularly in patients undergoing neoadjuvant chemotherapy. Some variability in immunohistochemistry results may be attributed to tissue fixation, antigen retrieval and interpretation of the stain result, rather than the biology of the tumor itself. The variability bias is important in the equivocal group $(2+)$ particularly in terms of treatment decision making. The objective of our study is to evaluate the heterogeneity in the expression of HER2 protein expression by immunohistochemistry for $2+$ immunohistochemistry cases in order to ascertain the extent of test result variation based on the tissue being tested (core-needle biopsies vs resection specimen (RS)). ${ }^{6}$

\section{Materials and methods}

Thirty-seven invasive breast carcinomas diagnosed on breast core-needle biopsies with HER2 scored as weakly positive (immunohistochemistry score $2+$ ), were selected from our pathology files from January to December 2005. The corresponding FISH results were obtained from our cytogenetics laboratory. FISH was performed at the time of initial diagnosis using PathVysion dual color probe (Vysis Inc.,
Downers Grove, IL, USA). FISH was scored as amplified, if the HER2 signals to chromosome 17 centromere signals were greater than 2.2 (Figure 1a) and nonamplified if the ratio is less than 1.8 (Figure 1b). The follow-up RS were reviewed and two additional tumor blocks were selected for retesting for HER2 by immunohistochemistry. Seventy-four tissue (74) blocks were examined for HER2 protein expression, by Ventana Pathway HER-2 (clone CB-11) antibody, cat number; 760-2694, using a protocol set by the manufacturer on the Benchmark XT; Ventana Medical System, Tucson AZ. ${ }^{7}$ FISH was repeated on a representative tumor resection block.

\section{Specimen Processing}

At our institution, core-needle biopsies are exposed to $10 \%$ neutral-buffered formalin for a minimum of $6 \mathrm{~h}$ prior to immunohistochemistry testing. The resection specimens are similarly exposed to formalin for a minimum of $6 \mathrm{~h}$. In addition, the
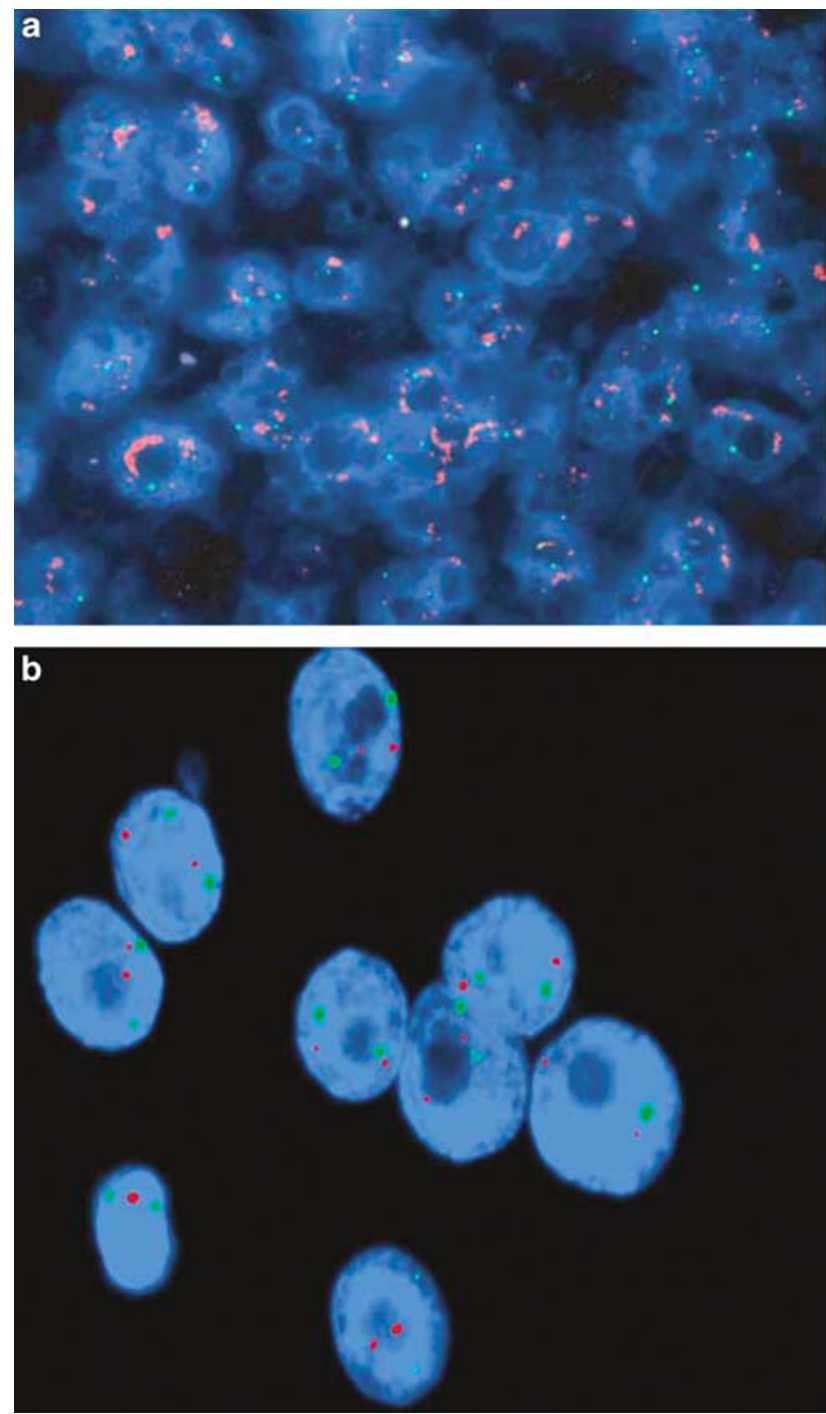

Figure 1 (a) HER2 amplified. (b) HER2 non-amplified. 
tissue blocks from RS are exposed to a $10 \%$ formalin-ethanol fixative (PENFIX ${ }^{\circledR}$, Richard Allan Scientific, MI, USA) for $2 \mathrm{~h}$ before they are exposed to $10 \%$ neutral-buffered formalin. ${ }^{8}$

HER2 scoring: The scoring was performed using the same guidelines as HercepTest ${ }^{\mathrm{TM}}$.

- 0-negative (no staining is observed or membrane staining is observed in less than $10 \%$ of tumor cells);

- 1+-negative (A faint/barely perceptible membrane staining is detected in more than $10 \%$ of the tumor cells);

- $2+$-positive (A weak to moderate complete membrane staining is observed in more than $10 \%$ of the tumor cells);

- 3+-positive (A strong complete membrane staining is observed in more than $10 \%$ of the tumor cells).

Heterogeneous staining is defined in our study when two different blocks showed different HER2 staining scores.

\section{Results}

\section{HER2 Results on Resection Blocks}

\section{Amplified group}

Strong protein expression was seen in 56\% (11/18) as evidenced by areas of $3+$ (Figure 2a) in both the blocks. Small areas of $2+$ were noticed within the same block in up to half of the cases (Figure 2b). $28 \%(5 / 18)$ remained $2+$. Two cases $11 \%(2 / 18)$ in this group showed negative staining $(0$ or $1+)$. $5.6 \%(1 / 18)$ showed heterogeneous staining pattern. In one case (case-9), there was no tumor left in one block, the other block showed immunohistochemical score $2+$ staining. There was focal area of 'no staining' or 'immunohistochemical score 0' among the strong positivity (case15) areas (Figure 2c). The immunohistochemical staining of resection blocks are shown in Table 1.

\section{Nonamplified group}

The most significant finding in this group is that none of the cases showed strong $3+$ staining

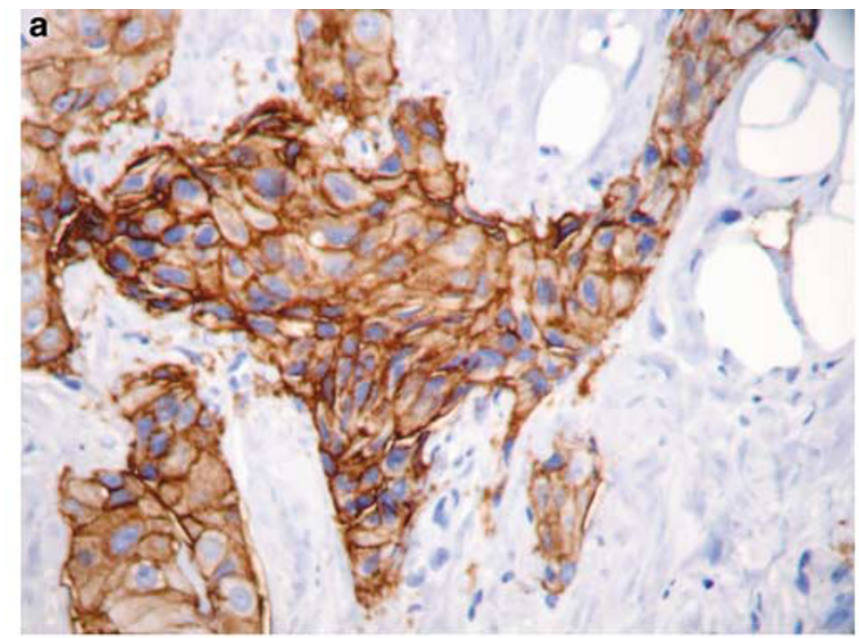

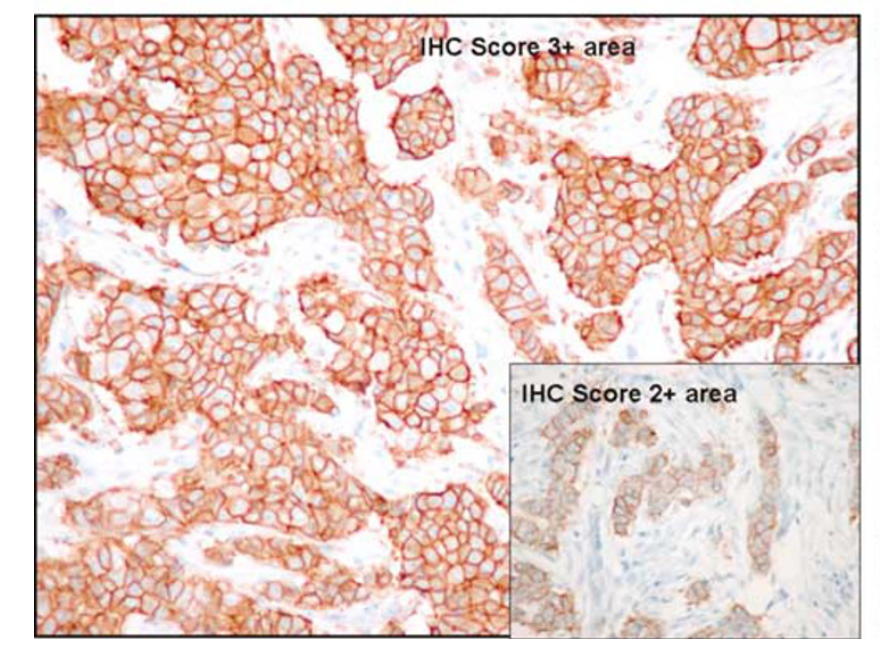

b

C

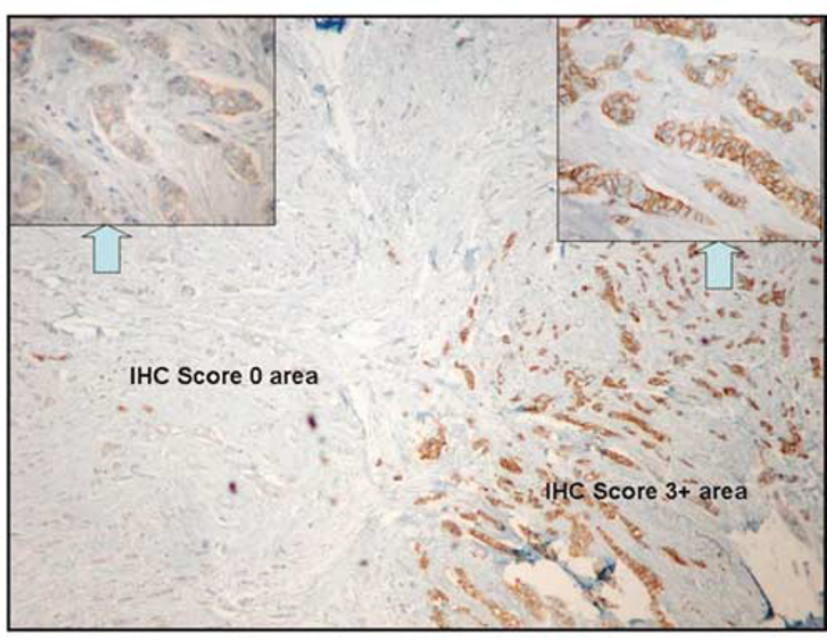

Figure 2 (a and b) Patterns of staining (pattern 1): IHC $3+$ predominantly with focal areas of IHC $2+$ (inset). (c) Patterns of heterogeneous staining IHC $3+$ areas with IHC '0' areas within the same tumor. 
Table 1 IHC results on RS blocks in the AMP group

\begin{tabular}{|c|c|c|c|c|c|}
\hline \multirow[t]{2}{*}{$\begin{array}{l}\text { Case } \\
\text { number }\end{array}$} & \multirow{2}{*}{$\begin{array}{c}\text { FISH } \\
\text { result } \\
\text { on } C N B\end{array}$} & \multicolumn{3}{|c|}{$\begin{array}{l}\text { IHC result on } \\
\text { RS blocks }\end{array}$} & \multirow[t]{2}{*}{$\begin{array}{l}\text { FISH result } \\
\text { on } R S \text { block }\end{array}$} \\
\hline & & Block-1 & Block-2 & Comments & \\
\hline 1 & Amp & $3+$ & $3+$ & Focal 2+areas & $>6.74$ \\
\hline 2 & Amp & $1+$ & $1+$ & & 1.17 \\
\hline 3 & Amp & $3+$ & $3+$ & Focal 2+areas & 4.40 \\
\hline 4 & Amp & $2+$ & $2+$ & & 2.43 \\
\hline 5 & Amp & $3+$ & $3+$ & Focal 2+areas & 1.35 \\
\hline 6 & Amp & $3+$ & $3+$ & & $>5.18$ \\
\hline 7 & Amp & $3+$ & $3+$ & Focal 2+areas & 6.76 \\
\hline 8 & Amp & $3+$ & $3+$ & & $>6.25$ \\
\hline $9^{\mathrm{a}}$ & Amp & - & $2+$ & & 1.18 \\
\hline 10 & Amp & $3+$ & $3+$ & & 5.40 \\
\hline 11 & Amp & $3+$ & $3+$ & & 1.95 \\
\hline 12 & Amp & $2+$ & $2+$ & & 2.99 \\
\hline 13 & Amp & $2+$ & $2+$ & & FAILED \\
\hline 14 & Amp & 0 & 0 & & 2.75 \\
\hline 15 & Amp & $3+$ & $3+$ & Focal' 0'areas & 1.51 \\
\hline $16^{\mathrm{b}}$ & Amp & $2+$ & $3+$ & & 5.42 \\
\hline 17 & Amp & $3+$ & $3+$ & & $>6.4$ \\
\hline 18 & Amp & $2+$ & $2+$ & Focal 2+areas & 2.89 \\
\hline
\end{tabular}

${ }^{\mathrm{a}}$ There is no tumor left in Block 1.

bCases with 'heterogeneous' staining.

Table 2 IHC results on RS blocks in the Nonamp group

\begin{tabular}{|c|c|c|c|c|}
\hline \multirow[t]{2}{*}{$\begin{array}{l}\text { Case } \\
\text { number }\end{array}$} & \multirow[t]{2}{*}{$\begin{array}{l}\text { FISH } \\
\text { result }\end{array}$} & \multicolumn{2}{|c|}{$\begin{array}{c}\text { IHC result } \\
\text { on RS blocks }\end{array}$} & \multirow[t]{2}{*}{$\begin{array}{l}\text { FISH result } \\
\text { on } R S \text { block }\end{array}$} \\
\hline & & Block-1 & Block-2 & \\
\hline 1 & Nonamp & 0 & 0 & 1.05 \\
\hline $2^{\mathrm{a}}$ & Nonamp & $2+$ & $1+$ & 1.00 \\
\hline 3 & Nonamp & $2+$ & $2+$ & 1.45 \\
\hline 4 & Nonamp & $2+$ & $2+$ & 1.04 \\
\hline 5 & Nonamp & $2+$ & $2+$ & 1.04 \\
\hline 6 & Nonamp & $2+$ & $2+$ & 0.99 \\
\hline 7 & Nonamp & $1+$ & $1+$ & 0.80 \\
\hline 8 & Nonamp & $0+$ & $1+$ & 1.13 \\
\hline 9 & Nonmp & $1+$ & $1+$ & 1.11 \\
\hline 10 & Nonamp & 0 & 0 & 1.04 \\
\hline 11 & Nonamp & $1+$ & $1+$ & 1.00 \\
\hline 12 & Nonamp & $2+$ & $2+$ & 1.20 \\
\hline $13^{\mathrm{a}}$ & Nonamp & $2+$ & $1+$ & 1.10 \\
\hline 14 & Nonamp & $2+$ & $2+$ & 2.04 \\
\hline 15 & Nonamp & $2+$ & $2+$ & 1.15 \\
\hline 16 & Nonamp & 0 & 0 & 1.39 \\
\hline 17 & Nonamp & $2+$ & $1+$ & 1.37 \\
\hline $18^{\mathrm{a}}$ & Nonamp & $1+$ & $2+$ & 1.33 \\
\hline 19 & Nonamp & $0+$ & $0+$ & FAILED \\
\hline
\end{tabular}

${ }^{\mathrm{a} C a s e s}$ with heterogeneous staining.

$0 \%(0 / 19)$. In all $42 \%(8 / 19)$ had score $0-1+, 37 \%$ (7/19) remained as $2+$ by immunohistochemistry. The immunohistochemical staining of resection blocks are shown in Table 2.

\section{Heterogeneous staining cases}

Heterogeneous staining was observed in five cases in both the groups. The immunohistochemistry scores were $1+$ (block 1), $2+$ (block 2 ) respectively
Table 3 Expression of HER2 in RS based on the FISH results of the corresponding CNB

\begin{tabular}{lcc}
\hline HER2 score & $\begin{array}{c}\text { Amplified (Amp) } \\
\text { group (n-18) }\end{array}$ & $\begin{array}{c}\text { Nonamplified (Nonamp) } \\
\text { group (n-19) }\end{array}$ \\
\hline $\begin{array}{l}\text { Negative (0 or } 1+) \\
\text { Equivocal (2+) }\end{array}$ & $11 \%(2 / 18)$ & $42 \%(8 / 19)$ \\
$\begin{array}{l}\text { Strongly positive } \\
(3+)\end{array}$ & $56 \%(11 / 18)$ & $37 \%(7 / 19)$ \\
$\begin{array}{l}\text { Heterogeneous } \\
(1+, 2+, 3+)\end{array}$ & $5.6 \%(1 / 18)$ & $0 \%(0 / 19)$ \\
\hline
\end{tabular}

in the nonamp group. In the amplified group, the immunohistochemistry scores were $2+$ (block 1 ), $3+$ (block 2). A comparison of immunohistochemistry staining of RS in the amp group and nonamp group are shown in Table 3.

FISH results on resection blocks

Amplified group In the amplified group, $22 \%$ (4/18) did not show amplification. One FISH test failed. Amplification was detected in $72 \%(13 / 18)$ cases (Table 1).

Nonamplified group In the nonamplified group, amplification was not detected in majority of the cases $89 \%(17 / 19)$. One case showed amplification. FISH test failed in one case (Table 2).

\section{Discussion}

HER2 is a member of the epidermal growth factor receptor family of protein RTKs that is important in initiating signal transduction pathways in normal and abnormal cells. ${ }^{3}$ HER2 is overexpressed in $15-20 \%$ of the invasive breast carcinomas. ${ }^{1,2,4-6}$ HER2 expression is an individual prognostic factor in predicting the aggressive behavior of the tumor as well as predicting the benefit from adjuvant therapy. Trastuzumab suppresses HER2 activity, thereby facilitating apoptotic cell death. Clinical trials have shown that relative risk of recurrence is decreased by $50 \%$ when trastuzumab is added to the adjuvant chemotherapy regimen in HER2 positive group of women. ${ }^{3}$ Therefore, it is important to assess the HER2 status accurately for patients who can benefit from targeted therapy. To detect HER2 status following methods was used-immunohistochemistry to evaluate the level of HER2 protein in invasive carcinomas and FISH to assess gene amplification. The immunohistochemistry methods use polyclonal or monoclonal antibodies including CB11, AO485, HercepTest $^{\mathrm{TM}}$ and TAB320. The FDA approved PathVysion FISH method uses dual color fluorescent probes that are complimentary to HER2 gene and the centromere of Chromosome 17, on which it is present. The number of each type of signals per cell determines if amplification has occurred. The amplification is defined by PathVysion as the ratio 
of HER2 gene copies to chromosome 17 equal to or greater than 2.0 per cell. ${ }^{3,9}$ The two methods have their own advantages and disadvantages. The immunohistochemistry method is less expensive, less cumbersome, with a shorter assay time. However the results may be affected by the processing and fixation methods. There is variability in the HER2 expression using different monoclonal and polyclonal antibodies, ranging from 26 to $42 \%$. The discrepancies in the published literature regarding increased expression levels of these different antibodies are attributed to the possibility of variations in tissue preparation and scoring criteria rather than the nature of these antibodies. ${ }^{6}$ FISH methods on the other hand are semiquantitative assays which are less subjective but are expensive. ${ }^{3,6}$

The comparison of the immunohistochemistry and FISH assays are extensively addressed in the literature. These studies have shown an excellent correlation with immunohistochemistry staining of $3+$ in which almost all showed gene amplification ranging from 89 to $100 \% .^{3,4,10-12}$ FISH nonamplified tumors with immunohistochemistry score of $3+$ is $1.2 \% .{ }^{9}$ Retrospective comparison of FISH amplification to the $2+$ score in studies have shown that $8-25 \%$ cases show amplification ${ }^{1,4,13}$ which explains that it is reasonable to subject the $2+$ cases to FISH assays to identify this small subset of patients who can benefit from trastuzumab therapy.

In clinical trials that have examined the benefit of trastuzumab monotherapy, a retrospective FISH analysis was performed on the tumor samples that were scored $2+$ or $3+$ on immunohistochemistry. A response of $35 \%$ was seen in the patients with FISH amplified group in comparison to a low $3 \%$ response in patients with FISH nonamplified group, which indicate that tumors that show HER2 positivity by immunohistochemistry and FISH assays respond to trastuzumab therapy in up to $50 \%$ cases. ${ }^{3,9,14}$ Similar results were seen in randomized phase III study to evaluate the efficacy of trastuzumab combined with chemotherapy by Slamon et al, found substantial increase in response rate in group with immunohistochemistry $2+$ or $3+$ with FISH amplified group than the immunohistochemistry $2+$ or $3+$ with FISH nonamplified group. ${ }^{15}$ In summary, all the clinical trials address the importance of HER2 biomarker status in clinical decision making.

The important question to be addressed which will be of great relevance for the clinicians and pathologists are the selection of the ideal archival tissue to perform the HER2 staining. Core-needle biopsies have been demonstrated by few studies to accurately show the expression of the prognostic markers including HER2 $2^{8,11,16}$ and are far more superior presumably because small biopsies fix more rapidly and uniformly than resection specimens. In an attempt to select the ideal specimen, we compared the expression of HER2 in core-needle biopsies and resection specimens in equivocal cases rather than all the cases. In our study, in the amplified group, a significant number of cases showed positive staining with immunohistochemistry score $3+$. Among these cases more than half of the cases showed areas of immunohistochemistry $2+$ within the same block. In our opinion the finding of positive $3+$ areas in the resection specimens might not be truly discordant with the coreneedle biopsies results. As compared to a small surface area of tumor within a core-needle biopsies, the resection specimens have a larger surface area of tumor with larger field of staining which might have revealed the immunohistochemistry $3+$ areas. On the other hand, due to the larger surface area of tumor, the intensity of staining is variable as noticed in one of the cases with areas of negative staining within strongly staining areas. In the nonamplified group, core-needle biopsies with $2+$ immunohistochemistry had no appreciable HER2 immunohistochemistry heterogeneity in the resection specimens that would have altered the patient treatment, which is confirmed by our cases showing no strong $3+$ staining. Heterogeneous staining is observed in both the groups, predominantly in the nonamplified group. However, the heterogeneity of staining in both the groups may not be of significant impact on clinical decision making. The heterogeneity and the variability of staining within the same block or in two different blocks is most likely due to biologic variation, although variable fixation may be a contributing factor.

To summarize, the core-needle biopsies sample is an excellent specimen for both immunohistochemistry and FISH testing. Amplification of HER2 in core-needle biopsies is associated with a substantial probability of finding strong staining in resection specimens, while no HER2 amplification of equivocal immunohistochemistry on core-needle biopsies is associated with $3+$ HER2 immunostaining on resection specimens.

\section{Acknowledgement}

We thank Kim McManus HT (ASCP, histotechnology specialist) for her technical assistance.

Presented in part as poster at San Antonio Breast Cancer Symposium, San Antonio, TX, USA (December 18-22), 2006.

\section{References}

1 Hammock L, Lewis M, Phillips C, et al. Strong HER2/neu protein overexpression by immunohistochemistry often does not predict oncogene amplification by fluorescence in situ hybridization. Hum Pathol 2003; 34:1043-1047.

2 Wolff AC, Hammond ME, Schwartz JN, et al. American Society of Clinical Oncology; College of American 
Pathologists. American Society of Clinical Oncology/ College of American Pathologists Guideline recommendations for human epidermal growth factor receptor 2 testing in breast cancer. Arch Path Lab Med 2007; 131:18-43.

3 Carlson RW, Moench SJ, Hammond ME, et al. NCCN HER2 testing in breast cancer task force. HER2 testing in breast cancer: NCCN task force report and recommendations. Natl Compr Canc Netw 2006;4(Suppl 3): S1-S22; quiz S23-S24.

4 Perez EA, Roche PC, Jenkins RB, et al. HER2 Testing in patients with breast cancer: poor correlation between weak positivity by immunohistochemistry and gene amplification by fluorescence in situ hybridization. Mayo Clin Proc 2002;77:148-154.

5 Diaz NM. Laboratory testing of HER-2/neu in breast carcinoma: an evolving strategy to predict response to targeted therapy. Can Control 2001;8:415-418.

6 Lebeaue A, Deimling D, Kaltz C, et al. HER-2/neu analysis in archival tissue samples of human breast cancer: comparison of immunohistochemistry and fluorescent in situ hybridization. J clin Oncol 2001; 19:354-363.

7 CB11, Tucson, Arz, Ventana Medical Systems Inc. [package insert].

8 Taucher S, Rudas M, Mader RM, et al. Prognostic markers in breast cancer: the reliability of HER2/neu status in core needle biopsy of 325 patients with primary breast cancer. Wien Klin Wochenschr 2004; 116:26-31.

9 Vogel CL, Cobleigh MA, Tripathy D. Efficacy and safety of trastuzumab as a single agent in first line treatment of HER 2 overexpressing metastatic breast cancer. J Clin Oncol 2002;20:719-726.

10 Yaziji H, Goldstein LC, Barry TS, et al. HER-2 testing in breast cancer using parallel tissue-based methods. JAMA 2004;291:1972-1977.

11 Wang S, Saboorian MH, Frenkel E, et al. Laboratory assessment of the status of HER-2/neu protein and oncogene in breast cancer specimens: comparison of immunohistochemistry assay with fluorescence in situ hybridization assays. Clin Pathol 2000;53:374-381.

12 Ridolfi RL, Jamehdor MR, Arber JM. HER-2/neu testing in breast carcinoma: a combined immunohistochemical and fluorescent in situ hybridization approach. Mod Pathol 2000;13:866-873.

13 Barrett C, Magee H, O’Boyle D, et al. Amplification of the HER-2 gene in breast cancers testing 2+ weak positive by HercepTest ${ }^{\mathrm{TM}}$ immunohistochemistry: false positive or false negative immunohistochemistry? Clin Pathol 2007;60:690-693.

14 Colbeigh MA, Vogel CL, Tripathy D. Multinational study of the efficacy and safety of humanized anti-HER 2 monoclonal antibody in women who have HER2 overexpressing metastatic breast cancer that has progressed after chemotherapy for metastatic disease. J Clin Oncol 1999;17:2639-2648.

15 PathVysion HER-2 [Package insert]. Downers Grove, Ill. Vysis Inc., 2001.

16 Jacobs TW, Siziopikou KP, Prioleau JE, et al. Do prognostic marker studies on core needle biopsy specimens of breast carcinoma accurately reflect the marker status of the tumor? Mod Pathol 1998;11: 259-264. 